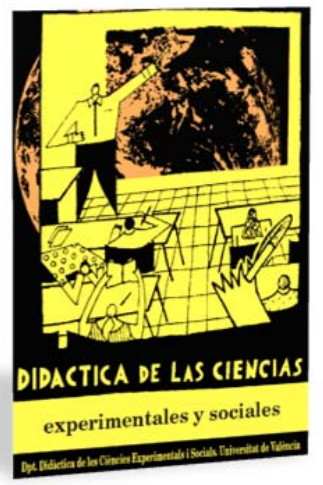

\title{
Entrelazando la Ética con las Ciencias Experimentales: una propuesta didáctica para la capacitación de profesores con la serie Breaking Bad
}

\section{Weaving Ethics with Experimental Sciences: a didactic proposal for teacher training with the TV series Breaking Bad}

DOI: $10.7203 / D C E S .34 .11478$

\author{
Irene Cambra Badii \\ Universidad de Buenos Aires - CONICET, irenecambrabadii@gmail.com \\ ORCID iD: https://orcid.org/0000-0003-1233-3243 \\ María Gabriela Lorenzo \\ Universidad de Buenos Aires - CONICET, glorenzoffyb@gmail.com \\ ORCID iD: https://orcid.org/0000-0002-9957-8392
}

RESUMEN: Cada vez es más evidente la necesidad de articular las cuestiones disciplinares con la Naturaleza de la Ciencia, las relaciones entre Ciencia, Tecnología y Sociedad, y la interrogación sobre aspectos éticos de la enseñanza y del conocimiento. Presentamos una investigación-acción a partir de una propuesta en base a la serie Breaking Bad para la capacitación de profesores de ciencias. Los resultados mostraron diferentes formas de concebir la ciencia, el trabajo científico y su enseñanza. Se desataca el surgimiento de situaciones éticamente controversiales en la escuela secundaria. La propuesta promovió el debate y la reflexión sobre una serie de hechos con un amplio impacto social, y, se facilitaron los procesos metacognitivos necesarios para la transformación de las prácticas educativas.

Palabras Clave: Naturaleza de la Ciencia, ética, series televisivas, propuesta didáctica, imagen de la ciencia.

ABstract: The need to articulate disciplinary issues with the Nature of Science, the relationship between Science, Technology and Society, and the interrogation on ethical aspects of teaching and knowledge is becoming more and more evident these days. In this article, we present an action-research consisting on a proposal for training science teachers based on the TV-series Breaking Bad. The results showed different ways of conceiving science, scientific work and its teaching. The rise of ethically controversial situations in secondary school is remarkable. The proposal encouraged debate and reflection on a series of events with broad social impact, and provided the necessary metacognitive processes for the transformation of educational practices.

KEYWORDS: Nature of Science, ethics, TV series, didactic proposal, image of science.

Fecha de recepción: febrero de 2018

Fecha de aceptación: marzo de 2018

Este trabajo fue realizado gracias al financiamiento otorgado por los siguientes proyectos: PIP 11220130100609CO (2014-2016); PICT-2015-0044 y UBACYT 20020130100592BA (2014-2017). 


\section{INTRODUCCIÓN}

El conocimiento científico es un bien social, aunque a veces quede definido de manera difusa o se desconozcan los procesos que originaron su construcción. Por eso, la enseñanza de las ciencias ${ }^{1}$ presenta diversos desafíos como la importancia de incluir aspectos disciplinares y pedagógicos (Shulman, 1986) y al contexto sociohistórico (Lorenzo y Farré, 2016; Ruiz y Solbes, 2013; Solbes y Traver, 1996, 2001; Solbes y Vilches, 1989, 2004), la necesidad de formación docente (Caena, 2011; Darling, 2006; Escudero, 2011; Timperley y Alton Lee, 2008, Wei, Darling y Adamson, 2010), la inclusión de nuevas tecnologías de la información y la comunicación (TIC) (Almerich, Suárez, Orellana y Díaz, 2010; Coll, Mauri y Onrubia, 2008; Law y Chow, 2008; Tearle, 2003), e incluso, el cambio de valores en la sociedad contemporánea (Gavidia, 2005) y la situación del sistema de enseñanza, ya sea de nivel universitario o secundario (Gibbs y Coffey, 2014), y las particularidades de enseñar en uno u otro contexto (Lorenzo, Farré y Rossi, 2013; Lorenzo, 2017).

En la práctica docente se imbrican variadas cuestiones que confluyen en el dictado cotidiano de clases. Además de la disciplina de base se requiere de los aportes de las ciencias sociales (historia de las ciencias, sociología, filosofía, entre otras) para la comprensión del conocimiento científico y de sus modos de producción. Por ejemplo, a través de la Naturaleza de la Ciencia (NdC)y las implicancias de la relación entre Ciencia, Tecnología y Sociedad (CTS) como de la posibilidad de interrogarnos sobre aspectos éticos de la enseñanza y del conocimiento. Es así que surge un nuevo campo de interés que entrelaza las nociones éticas con las prácticas educativas y de investigación de las ciencias experimentales. Esto implica preguntarnos por el modelo mismo de educación, cuestionando los valores dominantes, la responsabilidad de los investigadores respecto del conocimiento y sus formas de producción (Jiménez, García, Azcárate y Navarrete, 2015) y los límites en el uso del conocimiento científico, sus implicancias sociales y el impacto que puede tener para las generaciones futuras el uso de determinadas tecnologías.

Una vía productiva para la indagación reflexiva de las cuestiones éticas es la narrativa cinematográfica, como el cine o las series de TV (Astudillo, Rivarosa y Ortiz, 2012; Palacios, 2007; Petit y Solbes, 2015). Un ejemplo de gran impacto entre el gran público es la serie Breaking Bad (Cuadro I). Esta cuenta el derrotero de Walter White, un profesor de química de secundario que, cuando recibe el diagnóstico de cáncer terminal, se dedica a producir metanfetamina. La ansiedad por conocer el destino de White, quien adopta el seudónimo de Heisenberg para ocultar su identidad mientras se dedica a la fabricación y a la comercialización de la droga, hizo que la serie fuera una de las más vistas, generando récords de audiencia y cosechando excelentes críticas (Adalian, 2013; Ryan, 2012).

En este artículo presentamos una propuesta didáctica para un taller de profesores de ciencias y discutimos los resultados de su implementación pensando en sus posibilidades a futuro.

\section{MARCo TEÓRICo}

Este trabajo propone una articulación original entre la enseñanza de las ciencias, los contenidos disciplinares, el modelo CTS y el atravesamiento de la interrogación ética sobre el conocimiento científico y sus formas de producción.

A partir del modelo sobre el conocimiento didáctico del contenido (Shulman, 1986) se reconoció la importancia de poseer un tipo de conocimiento especial que le permita al docente transformar el contenido disciplinar en actividades de aprendizaje significativas para sus estudiantes. En dicho conocimiento se integran aspectos de la NdC promoviendo la contextualización y revisión permanente del conocimiento científico, sus enunciados y sus prácticas para no incurrir en la mera repetición de hechos científicos, leyes y teorías (Bell y Lederman, 2003).

\footnotetext{
${ }^{1}$ En este trabajo nos referiremos al grupo de disciplinas vinculadas a las ciencias experimentales con base en la química, la física y la biología, resumidamente como ciencias.
} 
Además, Solbes y Torres (2014, 2015) proponen analizar las cuestiones científicas y los conflictos de los científicos en sus producciones en relación con sus implicaciones sociales, denominándolas cuestiones sociocientíficas. En esta línea, Vieira y Nascimento (2007), Solbes y Vilches (2004), Ruiz y Solbes (2013) y Lorenzo y Farré (2016) destacan la necesidad de introducir discusiones en la didáctica de las ciencias puntualizando como eje principal el enfoque CTS, ya que se halla en relación con la NdC y la articulación del conocimiento científico con sus condiciones sociales de producción, para favorecer la participación ciudadana en los procesos democráticos de toma de decisión a partir de la alfabetización científica y tecnológica (Membiela, 1997).

Por otro lado, la sociedad posee una imagen negativa de la ciencia, considerada aburrida, difícil y responsable de algunos de los grandes problemas de nuestro tiempo (Solbes y Traver, 2001; Solbes, Monserrat y Furió, 2007). De tal forma, la enseñanza de las ciencias conlleva el replanteo de los valores y de las finalidades del conocimiento científico (Solbes, 1999), considerando su utilidad y el análisis de las cuestiones éticas implicadas en cada producción de conocimiento, los valores que la sustentan, el uso que le será conferido y la coherencia entre un aspecto y otro.

Ahora bien ¿cómo conceptualizamos a la ética y qué relación guarda con la enseñanza de las ciencias en este nuevo campo en construcción?

La pretensión de incorporar aspectos éticos en la enseñanza tiene como objetivo proporcionar instrumentos y claves relevantes para la toma de decisiones en un proceso científico que involucre a la sociedad en su conjunto. Considerando la naturaleza intrínsecamente moral de la educación (Hansen, 2001, Jackson, Boostrom y Hansen, 2003), surge la necesidad de diferenciar moral y ética. La moral comprende al conjunto de normas presentes en una sociedad, que pueden ser tanto explícitas (leyes, normativas, códigos de ética) como implícitas, a las cuales considera mayoría en función de códigos y saberes compartidos, pero no necesariamente legislados por escrito (Bolívar, 2005) que cambian según la época, el grupo y las condiciones sociales. A la posibilidad de reflexionar sobre ese conjunto de normas lo denominamos ética, según principios universalizables en función de la condición humana. La ética, como reflexión crítica de segundo orden sobre los valores o comportamientos previos, proporciona razones que justifican o no las acciones, analizando los comportamientos morales; explica, desde patrones de generalidad, la experiencia moral humana y prescribe los modos de comportamiento justificables.

Dado que cada actividad profesional es diferente, es necesario atender a los valores, objetivos y hábitos propios de cada uno. En el caso de la didáctica de las ciencias, se incluirían cuestiones relativas a la reflexión sobre el conocimiento científico, sus alcances y sus límites, tanto desde la perspectiva del docente como del investigador.

Desde esta perspectiva, la capacitación de los profesores de ciencia debe alejarse del dictado prescriptivo para tomar como base el modelo de enseñar enseñando (Lorenzo, 2012). (Lorenzo, 2012) con el fin de poner en acto una propuesta didáctica particular para la enseñanza de contenidos disciplinares concretos que incluyan los aportes de los modelos CTS y de las disciplinas metacientíficas. Así, por un lado, los profesores aprenden participando de la actividad, repensando sus propias prácticas docentes e integrando nuevas formas de enseñar con materiales teóricos y didácticos para transformar las prácticas actuales. Y por otro, el diseño pensado como una unidad didáctica en cascada facilita su adaptación y aplicación en sus propios contextos. Además, se favorece la exploración de la duplicidad de roles: como aprendices y como docentes capaces de analizar la enseñanza tanto ajena como propia, enriqueciendo sus propios conocimientos durante el proceso. En esta ocasión se decidió incorporar la interrogación ética, hasta ahora ausente en este tipo de capacitaciones. Para hacerlo, elegimos las imágenes visuales (Ortega, 2002) y en particular las series como medios privilegiados (Grilli, 2015, 2016); ya que, permiten conectar los avances científico-tecnológicos con aspectos sociales repercutiendo positivamente en la enseñanza de las ciencias (Perales y Vílchez, 2005; Pro-Bueno y Ezquerra, 2005; Zúñiga, Leiton y Naranjo, 2014). 
El cine constituye un recurso flexible capaz de complementar los métodos docentes clásicos (Dark, 2005) por la posibilidad de exposición de núcleos interesantes (o controversiales) del conocimiento científico (García Borras, 2008, 2011; Sierra, 2007; Petit y Solbes, 2016) al mostrar el lado humano de los científicos, posibilitando el debate sobre la supremacía del género masculino en la ciencia (Camacho, 2018) y de la imagen de los científicos locos inmortalizados en las películas de terror (Pujalte, Adúriz-Bravo y Porro, 2015).

Las imágenes y las historias relatadas aportan elementos que generan entusiasmo, a la vez que amplían el conocimiento conceptual y fomentan las capacidades críticas de los estudiantes. También posibilita el diseño de prácticas educativas que promueven la crítica, la observación, la reflexión e incluso la investigación (García Borrás, 2006). Una escena cinematográfica puede actuar como elemento motivador, para introducir de cierta discusión y al mismo tiempo, como detector de preconcepciones (Cubero, 1989). Además, el cine puede servir para el desarrollo del espíritu crítico y la actitud científica (Guisasola y Morentin, 2007).

Dentro de la narrativa cinematográfica, las series representan un universo complejo, cuyo alto índice de consumo a nivel mundial evidencia su gran impacto social. Las series son narrativas que promueven valores y naturalizan determinados comportamientos presentados por los personajes. En el caso Breaking Bad el protagonista es un antihéroe (Aguado, 2017) con lo cual la propuesta ética de la serie (López y Nicolás, 2015) exige un posicionamiento del espectador más allá de la identificación empática con el protagonista. Si bien, esta serie fue utilizada como herramienta educativa, para cuestiones de comunicación, en relación con las narrativas transmedia (Jenkins, Ford y Green, 2013; Ferrareli, 2015), en las relaciones humanas (Mateus y Chávez, 2014), para la introducción de conceptos específicos, como quiralidad y enantiomería (Torre y García, 2014) o para despertar el interés del estudiante (Ramos y Silva, 2017); en este trabajo desarrollamos una implementación original que se detalla a continuación.

\section{Metodología}

Se presenta un estudio exploratorio descriptivo sobre el diseño de una intervención didáctica y su posterior implementación en un taller de capacitación de profesores, con el fin de evaluar sus resultados y realizar los ajustes necesarios para futuras intervenciones. Se busca contriubuir a una investigación educativa críticamente comprometida para la transformación de las prácticas convencionales a la vez que genere nuevo conocimiento (Murillo e Idalgo, 2017).

Se enmarca, en la perspectiva de la investigación-acción (Nussbaum 2017) y propone una recogida de datos naturales desde una aproximación cuasietnográfica por ser la coordinadora del taller una de las investigadoras. De acuerdo con los objetivos se consideró la potencialidad del instrumento para la detección de las concepciones de los docentes sobre: las modalidades de enseñanza de la química, la imagen de ciencia y el uso del conocimiento científico. Los datos obtenidos de las distintas fuentes (registros escritos, audiograbaciones, observaciones) fueron triangulados entre sí.

\subsection{Diseño e implementación de la propuesta didáctica para taller de capacitación docente}

Los propósitos que guiaron el diseño de la propuesta didáctica, con el fin de promover una actualización integral de los profesores de ciencia a través de una experiencia vivencial son:

- Reflexionar sobre la inclusión del cine y las series en la enseñanza de las ciencias.

- Proponer formas de articulación conceptuales y prácticas para la interrelación entre las ciencias y la interrogación ética sobre los límites en el uso del conocimiento científico, la utilidad del conocimiento científico, la responsabilidad de las personas que hacen ciencia. 
- Considerar los interrogantes respecto de la necesidad de una formación ética para todos los docentes.

Para el diseño de la intervención se seleccionaron cuatro fragmentos de la serie Breaking Bad con una duración de entre 1 y 7 minutos, aplicando los criterios de accesibilidad desde el punto de vista tecnológico, su calidad cinematográfica, la temática abordada y el interés despertado por la obra. Los fragmentos fueron separados con el programa Adobe Premiere e incrustados como hipervínculos en la presentación de PowerPoint preparada especialmente para el taller.

CUADRO 1. Ficha técnica de la serie

Títule original: Breaking Bad (TV Series)

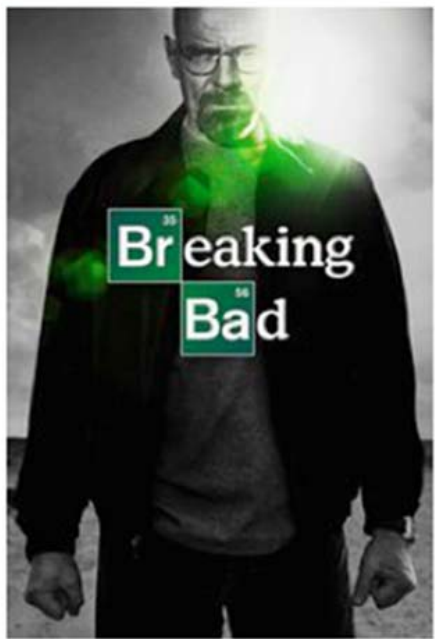

Peter Reniers

Años: 2008-2013

Duración de cada episodio: 45 minutos

Pais: Estados Unidos

Dirección: Vince Gilligan (Creator), Michelle MacLaren, Adam Bernstein, Vince Gilligan, Colin Bucksey, Michael Slovis, Bryan Cranston, Terry McDonough, Johan Renck, Rian Johnson, Scott Winant, Peter Gould, Tricia Brock, Bronwen Hughes, Tim Hunter, Jim McKay, Phil Abraham, John Dahl, Félix Enríquez Alcalá, Charles Haid, Peter Medak, John Shiban, David Slade, George Mastras, Thomas Schnauz, Sam Catlin

Guión: Vince Gilligan, Peter Gould, George Mastras, Sam Catlin, Moira Walley-Beckett, Thomas Schnauz, Gennifer Hutchison, John Shiban, J. Roberts, Patty Lin

Música: Dave Porter

Fotografia: Michael Slovis, Reynaldo Villalobos, Nelson Cragg,

Reparte: Bryan Cranston, Aaron Paul, Anna Gunn, Dean Norris, Betsy Brandt, RJ Mitte, Bob Odenkirk, Jonathan Banks, Giancarlo Esposito, Steven Michael Quezada, Jesse Plemons, Christopher Cousins, Laura Fraser, Matt Jones, Charles Baker, Michael Shamus Wiles, Lavell Crawford, Ray Campbell, Krysten Ritter, Ian Posada, Carmen Serano, Emily Rios, Tina Parker, Mark Margolis, Robert Forster, Caleb Landry Jones, Jeremiah Bitsui, David Costabile, Dale Dickey, Jere Burns, Steven Bauer, Daniel Moncada

Productora: Emitida por AMC; Gran Via Productions / High Bridge Productions / Sony Pictures Television

Género: Serie de TV. Thriller. Drama | Comedia negra, Crimen. Drogas. Enfermedad. Policíaco. Familia

Sinopsis: Serie de TV (2008-2013). 5 temporadas. 62 episodios. Tras cumplir 50 años, Walter White (Bryan Cranston), un profesor de química de un instituto de Albuquerque, Nuevo México, se entera de que tiene un cáncer de pulmón incurable. Casado con Skyler (Anna Gunn) y con un hijo discapacitado (RJ Mitte), la brutal noticia lo impulsa a dar un drástico cambio a su vida: decide, con la ayuda de un antiguo alumno (Aaron Paul), fabricar anfetaminas y ponerlas a la venta. Lo que pretende es liberar a su familia de problemas económicos cuando se produzca el fatal desenlace. (FILMAFFINITY)

Premios: 2014: 5 premios Emmy, incluyendo mejor serie dramática. 2013: 2 premios Emmy: Mejor serie drama y mejor actriz secundaria (Anna Gunn); Globos de Oro: Mejor serie de TV (Drama), Mejor actor. 2012: Emmy: Mejor actor secundario (Aaron Paul). 2010: 2 premios Emmy: Mejor Actor y Actor secundario (Aaron Paul). 2009: 2 premios Emmy: Mejor Actor y Edición. 2008: 2 premios Emmy: Mejor Actor y Edición.

Fuente: Filmaffinity

El taller se organizó en torno a 4 módulos con una duración total de 4 horas según el siguiente esquema: 


\section{Módulo 1: Presentación del taller}

El taller se inicia informando a los asistentes la modalidad de trabajo y enunciando las pautas de evaluación para su acreditación formal (producción grupal escrita durante el taller). Luego, se brinda un detalle general de la serie a modo de introducción.

Los asistentes se distribuyen en pequeños grupos, para participar de un debate organizado en dos etapas: 1) al interior de cada pequeño grupo, con la elaboración de un escrito. 2) con la puesta en común y debate en plenario del grupo con la coordinación del docente a cargo del taller cuya función es alentar el debate aportando preguntas específicas y aportando información.

\section{Módulo 2: Los contextos para la enseñanza de la ciencia}

\section{Objetivos}

- Analizar y comparar las modalidades de enseñanza de Walter White en el contexto formal (escuela secundaria) e informal (producción de droga con su ex alumno en una carretera estadounidense).

- Puntualizar distintas consideraciones respecto de la imagen de la ciencia que subyacen en los fragmentos, siguiendo las indicaciones del Cuadro II.

CUADRo 2. Descripción del módulo dos

Fragmento I. Clase de química en la escuela secundaria. Temporada 1, Episodio 1 (Piloto), minutos 7:20 hasta 8:59

Walter trata de llamar la atención de sus estudiantes, quienes están sentados en sus pupitres y lo miran aburridos. Explica: "la química es el estudio de la materia. Pero yo prefiero verla como el estudio de los cambios. Analicen esto: los electrones cambian su nivel de energía (enciende el mechero). Las moléculas cambian sus enlaces (arrojando distintas sustancias al fuego, que va cambiando de color). Los elementos se combinan y se transforman en compuestos. Esa es la vida misma ¿verdad?"

Los alumnos no le responden ni le prestan atención. De pronto Walter nota que una pareja de estudiantes enamorados está cuchicheando en el fondo del salón, envía al joven al asiento que le corresponde y, con expresión de enojo, pide a los estudiantes que abran el libro: "Enlaces iónicos, capítulo 4".

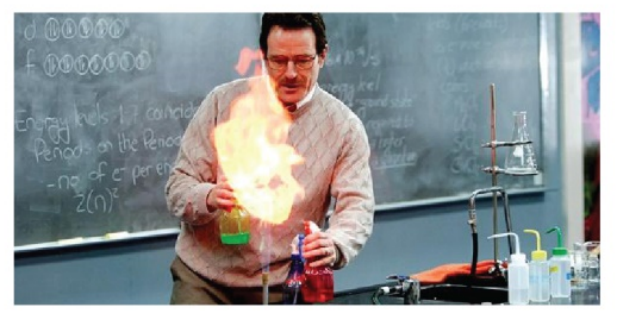

Permite analizar la modalidad de enseñanza expositiva y el escaso interés generado en los estudiantes.

Preguntas guía para el debate:

- ¿Cuál sería el modelo de enseñanza de Walter?

- ¿Qué dificultades aparecen en el desarrollo de la clase?

- ¿Cuál es la imagen de la ciencia que transmite este fragmento? 
Fragmento II. Producción de metanfetaminas en el desierto gracias a los conocimientos de Química de Walter White. Temporada 1, Episodio 1(Piloto), minutos 40:01 hasta 43:04.

Walter con su antiguo alumno, Jesse, están en el desierto para cocinar metanfetamina. Walter dirige la actividad en el laboratorio montado adentro de una camioneta, mientras Jesse lo asiste en distintas tareas, algunas veces torpemente. Así, obtienen cristales de metanfetamina (Torre-Fernández y García-Granda, 2014). de una pureza tal que Jesse no había visto nunca, y exclama: "Señor White, iusted es un artista! ¡Esto es arte!", a lo que Walter responde: "Bueno, gracias, pero es química básica".

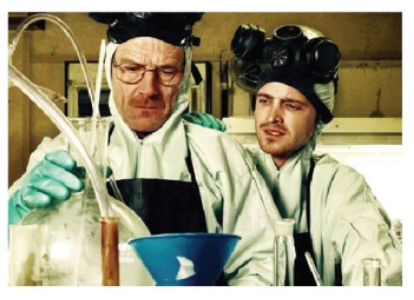

Permite analizar la actividad científica y el modelo de enseñanza tutor-aprendiz propia de las actividades de investigación. Preguntas guía:

- ¿Cuál es el rol que desempeña Walter White? ¿y Jesse?

- ¿Cuáles son las reacciones de su antiguo estudiante mientras le enseña los procesos químicos, y con la obtención del producto final?

- ¿En qué se asemeja y en qué se diferencia el modelo de enseñanza de este fragmento con el anterior?

- ¿Cuál es la imagen de la ciencia que transmite este fragmento?

Fuente: elaboración propia

Estas breves secuencias cinematográficas ponen en evidencia diferentes modelos de enseñanza con los cuales los participantes pueden identificarse o diferenciarse. Además, permite profundizar en la relación investigador científico-docente y reflexionar sobre el tipo de conocimiento que se requiere para desempeñar cada uno de esos roles. En el segundo fragmento, se observa a Walter White entusiasmado con el desarrollo del procedimiento químico, experto en su materia, y capaz de tolerar las distracciones o equivocaciones de su antiguo estudiante. El conocimiento químico y el conocimiento pedagógico aparecen claramente diferenciados, resultando evidente que Walter es, ante todo, un científico y no necesariamente un buen docente, salvo en esta circunstancia donde el proceso realmente lo entusiasma.

\section{Módulo 3: Los límites en el uso del conocimiento}

Objetivos

- Proponer articulaciones conceptuales entre la ciencia y las cuestiones éticas.

- Indagar concepciones relativas a los límites del conocimiento científico y su utilización.

- Debatir sobre la responsabilidad de Walter White, como químico y como profesor, en representación de la amplia comunidad de científicos y docentes. 
Fragmento III. Fabricación de una batería en el desierto. Temporada 2, Episodio 9 (Cuatro días fuera), minutos 34:17 hasta 40:43.

Walter y Jesse deciden ir al desierto a producir metanfetamina alejados de la ciudad. Cuando quieren regresar, se dan cuenta que la camioneta se quedó sin batería en medio del desierto, y Walter enseña a Jesse a construir una con los elementos que tienen disponibles.

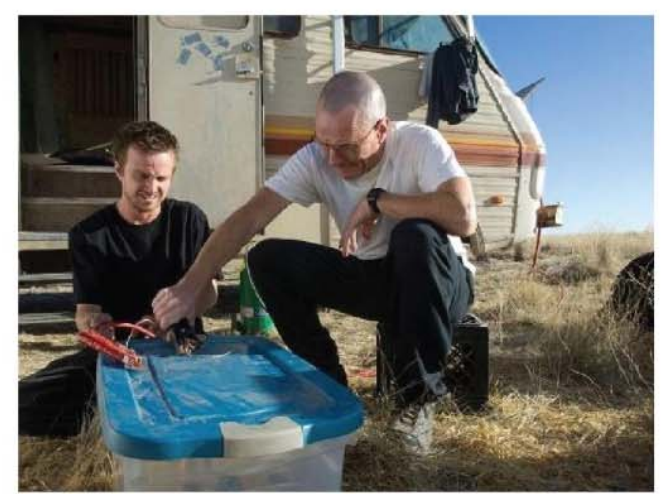

La elección de este fragmento tiene relación con la posibilidad de ver la potencia del conocimiento para la resolución de problemas cotidianos. En este caso, saber cómo armar una batería les da la posibilidad de no morir aislados en el desierto.

Preguntas guía:

- ¿Qué ideas aparecen en este fragmento sobre el conocimiento científico?

- ¿Cómo se articula el conocimiento con la solución a un problema concreto?

- ¿Cuál es la reacción del antiguo estudiante frente al procedimiento que realiza Walter?

- ¿Qué concepción de ciencia subyace en este fragmento?

Fragmento IV. Temporada 1, Episodio 6 (Loco puñado de nada), minutos 39:51 hasta 44:00.

Walter (con el seudónimo Heisenberg) acude a la oficina de uno de los distribuidores de metanfetamina para vengarse de la golpiza que le propinaron a Jesse. Intenta venderles una bolsa de droga pero los cristales son fulminato de mercurio $y$ produce una explosión.

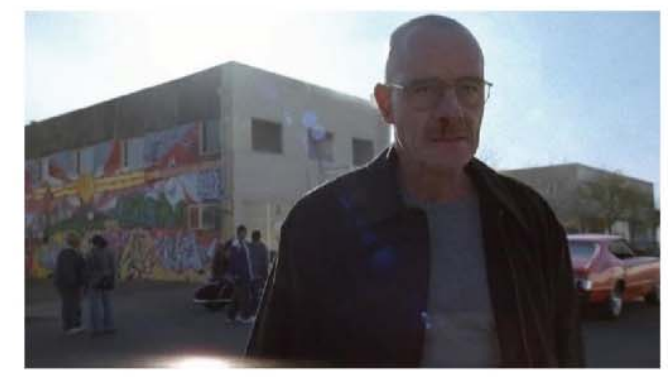

Este fragmento actúa como contraejemplo del anterior proponiendo un uso negativo (o no) del conocimiento.

Preguntas guía:

Idem Fragmento III

¿Qué piensan sobre lo que hizo Walter White/Heisenberg?

Fuente: elaboración propia 
Estos últimos fragmentos plantean dos posibilidades antagónicas en el uso del conocimiento, lo que facilita la indagación y explicitación de las concepciones de los participantes a partir del planteo de situaciones dilemáticas desde una perspectiva ética. Si en el desierto el conocimiento de Walter les permitió armar una batería para poder regresar, el cuarto fragmento (existen numerosos episodios en la serie en los cuales White utiliza su conocimiento de manera cuestionable, produciendo veneno o bombas caseras), muestra que el conocimiento también puede ser utilizado para dañar, ya sean daños materiales a una propiedad, como hiriendo a las personas que están en el lugar de la explosión.

\section{Módulo 4: Cierre del taller}

Finalmente, se plantea una revisión de toda la actividad promoviendo la discusión colectiva coordinada por el docente a partir de la enunciación de las preguntas como las siguientes: ciencia?

¿Cuál es la imagen de ciencia que subyace en la serie? ¿Y la de las personas que hacen

¿Cómo podría trabajar con sus estudiantes la conceptualización sobre:

- ¿ ¿Los medios y los fines para la construcción de conocimiento científico?

- ¿Las aplicaciones y usos del conocimiento?

¿Cuál es la responsabilidad del científico frente a la sociedad?

¿Cuál es la responsabilidad del docente?

Implementación: Con este diseño se implementó un taller en el marco de un congreso para profesores de química realizado en 2017 en la ciudad de Buenos Aires, bajo el nombre La química en la tele y viceversa: el caso Walter White de la serie Breaking Bad, en el cual participaron 40 docentes que se desempeñaban en los niveles secundario y universitario. Dado el carácter exploratorio de la investigación que no perseguía establecer correlaciones entre variables de género, edad o nivel educativo, sino documentar las concepciones docentes en un sentido amplio, no se describe la composición de los perfiles de la muestra de participantes.

Evaluación de la implementación: Con el fin de establecer los alcances de la actividad, sus puntos positivos y los aspectos a ser mejorados para futuras intervenciones, se recopiló la información vertida por los docentes en sus producciones escritas durante el taller. La Imagen 1 se presenta a modo ilustrativo. Los datos se complementaron con las desgrabaciones de los audios recogidos durante los debates plenarios y la observación no participante de una de las autoras.

La información recolectada fue analizada siguiendo el paradigma cualitativo, que incluye en su posición epistemológica una perspectiva más extensiva, centrada en el sentido, en la comprensión y en el significado (para la cual hemos tomado los aportes fundamentales de Taylor y Bogdan, 1992). Analizamos las producciones grupales de los participantes desde una perspectiva compleja, incluyendo lo singular de cada discurso, los detalles de sus dichos y los contextos a los cuales se articulan, mediante un análisis de contenido. Esta técnica permite estudiar y analizar cualquier forma de comunicación de una manera objetiva y sistemática, por otra parte, posibilita "realizar inferencias válidas y confiables de los datos con respecto a su contexto" (Hernández, Fernández y Baptista, 2006, p. 293). 
IMAGEN 1. Registros de los participantes al taller

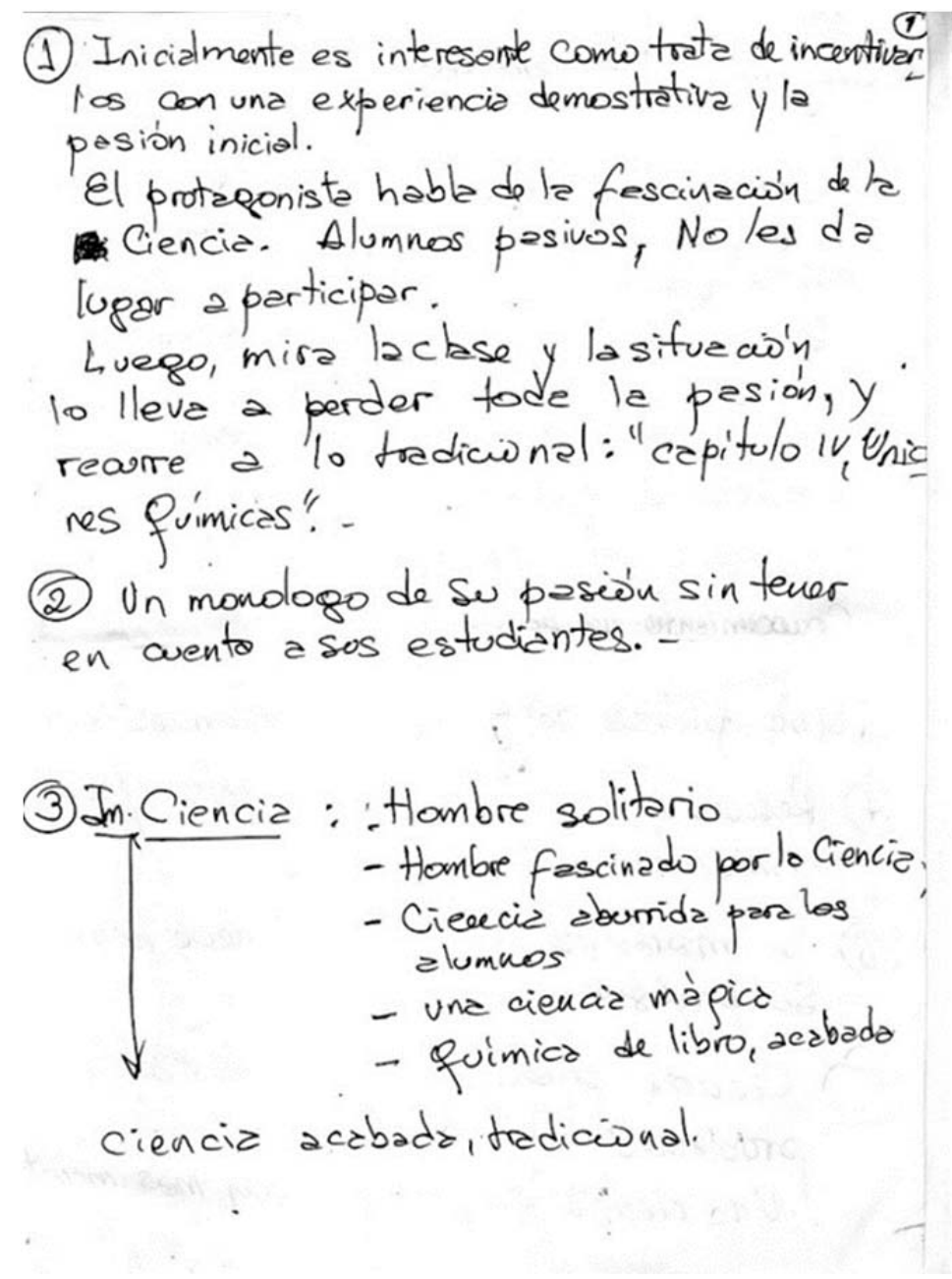

\section{RESUltadOS Y DiscuSióN}

Desde una primera aproximación los resultados mostraron: 1) la pertinencia de las categorías incluidas en el diseño de la propuesta por su potencialidad para entrelazar las cuestiones éticas con las prácticas científicas y de enseñanza de la ciencia; y 2) se evidenciaron ciertas inquietudes de los docentes, no previstas en el diseño original, que retroalimentan la discusión y nos ofrecen la oportunidad de revisar y mejorar la propuesta didáctica.

A continuación se presentan y discuten algunos comentarios de los participantes utilizando el entrecomillado y en cursiva, que sirven como ejemplos para el análisis de las categorías propuestas en el diseño, descriptas para los módulos 2 y 3.

\subsection{Análisis de la modalidad de enseñanza del Prof. White}

En todos los grupos se cuestionó la actuación de Walter en las escenas mostradas. Lejos de ver sus modalidades de enseñanza como opuestas, enfatizaron los puntos en común entre ambas situaciones: él es quien posee el conocimiento que es visto como complejo y difícil, y tiene algunas dificultades para la enseñanza de la química, aunque no en su práctica. En la discusión en plenario señalaron: "se nota una gran emoción y un gran entusiasmo por la química, pero no por los procesos de aprendizaje de la química". La clase en la escuela secundaria fue considerada "únicamente demostrativa, con el objetivo de llamar la atención y producir el interés de los estudiantes mediante una demostración vistosa, con colores”. Y que Walter la organiza bajo una 
modalidad expositiva ya que "no plantea preguntas y tampoco hace partícipe a los estudiantes". Los participantes señalaron que el ensayo de la llama, si bien es atractivo visualmente, podría ligarse a lo mágico, ya que, el Prof. White no incluye la explicación de los fundamentos teóricos para comprenderla.

Todos los participantes notaron la disposición espacial de la clase e hicieron énfasis en la imposibilidad de los alumnos de participar en la actividad de laboratorio planteada como mostrativa por el docente. Walter no daba la palabra a los estudiantes ni planteaba preguntas, pero tampoco explicaba lo que estaba haciendo. Los participantes señalaron: “el espacio y el lenguaje marcan una distancia del profesor con sus estudiantes".

El corte repentino de la clase al ver a una pareja de enamorados distraídos en el fondo y el regreso abrupto al libro de química, abandonando las actividades del laboratorio, fue uno de los aspectos más criticados: "los lleva al libro como un castigo", "lo domina la emoción al ver que no lo escuchan, no recupera la atención de los estudiantes y termina la actividad abruptamente".

Otros aspectos mencionados con menor frecuencia se referían a las pocas posibilidades de éxito de la modalidad demostrativa de la clase; y las posibilidades de las actividades prácticas como elemento disparador. Además, notaron que en la pizarra aparecen contenidos de química que no corresponden a la explicación verbal del profesor, haciendo presentes los recursos didácticos a los que recurren los docentes durante sus clases y la necesidad de coherencia entre ellos.

Se destaca la gran empatía mostrada por los participantes con el entusiasmo de Walter por la química. Con el primer fragmento, los docentes destacaron que Walter "intenta transmitir su alegría, pero cuando escucha las respuestas desencantadas de los estudiantes se desilusiona". No puede generar el interés de los estudiantes más allá de sus conocimientos: "uno puede saber mucho, pero si los estudiantes no prestan atención... no sirve de nada”. También, señalaron que Walter no tiene una conexión afectiva con los estudiantes. Da la clase, pero no se dirige a estos estudiantes en particular, de hecho parece que hablara como si no estuvieran allí.

Otro asunto mencionado fue el vínculo tutor-aprendiz: "Walter está motivado y así le enseña a Jesse", "hay cierta complicidad entre ambos, algo en común, una conexión”. Esto responde a otra faceta docente de Walter más próxima a lo que ocurre en los laboratorios de investigación durante la formación de becarios y tesistas, ya que se establece una relación más cercana en la que se comparte un interés común.

También resaltaron la reacción de sorpresa de Jesse, comparando los cristales de metanfetamina obtenidos con el arte, tanto por el valor que tienen para él los niveles de pureza de la droga como porque "no puede creer que sea su antiguo profesor, aquel docente aburrido". Esto muestra que más allá de tediosas explicaciones teóricas, el conocimiento de ciertas técnicas propias del laboratorio de química, proporcionan herramientas para la elaboración de un producto concreto, que en este caso particular, representa un valor social y económico para los interesados.

\subsection{Análisis de la imagen de ciencia}

A lo largo de los distintos fragmentos, fueron surgiendo diferentes concepciones respecto a la imagen de ciencia que subyacen en la serie. Entre los comentarios se encontraron los siguientes: “ $e l$ conocimiento de la Química parece ser un conocimiento acabado, imposible de repreguntar, espectacular, ligado a lo mágico. Es el docente quien define qué es la química, es un contenido cerrado que está en un libro, no es ni hipotética, ni provisoria, ni incompleta...”. O, refiriéndose a la modalidad de la clase del profesor "presenta una imagen distorsionada de lo que es enseñar química".

Asimismo, indicaron que, en líneas generales, el científico retratado en Walter insiste con algunos de los prejuicios y estereotipos de los hombres dedicados a la ciencia: seres solitarios, fascinados por un conocimiento complejo pero que termina siendo aburrido para los estudiantes.

En el fragmento II la imagen de la ciencia continúa ligada a un contenido puntual. Los participantes se preguntaron: “¿la ciencia sigue siendo aquello que se posee como conocimiento? 
¿La química es como seguir los pasos de una receta?” Reconocieron que para Walter no hubo un conocimiento nuevo, sino la manifestación de un "saber-hacer" en el laboratorio que le permite obtener productos especialmente buenos. Esta visión que correspondería a una imagen de ciencia normal desde una perspectiva kuhniana.

Los participantes distinguieron los diferentes roles de Walter como científico y como profesor, y destacaron su motivación, su entusiasmo y su nivel de pericia durante el proceso de síntesis y cristalización de la droga. Subrayaron: "se le ve en una actitud completamente diferente a la escena de la escuela, porque está haciendo lo que le gusta: química", "se le ve trabajando como un profesional, interviniendo con su antiguo estudiante sólo cuando él comete errores”.

\subsection{Análisis sobre el uso del conocimiento científico}

Los fragmentos III y IV mostraron otras vertientes de la representación sobre el conocimiento químico y su enseñanza. Además de los conocimientos teóricos y prácticos necesarios para la fabricación de una batería, sólo con los materiales que tenían en la camioneta, los participantes destacaron: "la disciplina misma parece como un caballero salvador, que los salva frente a las adversidades". Sin embargo, "no es algo mágico como en la escena de la escuela. Aquí aparece visible un proceso. Walter no tiene las respuestas desde el inicio sobre lo que hay que hacer para salvarse, e incluso cuando fabrica la batería no sabe si va a funcionar”. Esto implica, a diferencia de la discusión del fragmento I, que la imagen de la ciencia no radica en un conocimiento acabado, sino en una elaboración constante, en un proceso dinámico, con aciertos y errores, tal como mencionaron los participantes: "Walter elabora una hipótesis, realiza un intento que quizás no resulte. Pero la ciencia sigue siendo empírica”.

Más allá de esto, la ciencia continúa siendo representada como algo incomprensible para el lego, sigue siendo un conocimiento que posee Walter, quien evidentemente tiene el poder, y relega a Jesse a un lugar de asistente. A pesar de ello, reconocieron la dimensión de la creación colectiva del conocimiento: "Walter no podría haber construido la batería sin Jesse: no sólo por la cuestión técnica, sino porque no se le había ocurrido la idea. La ciencia no es un proceso solitario”.

Por otro lado, los participantes se refirieron a las diferentes concepciones de la química de Walter y de Jesse. Mientras para el experto predomina un pensamiento lógico para enfrentar los problemas, para el novato persiste cierto pensamiento mágico "todo es posible” y desde esta perspectiva aporta creatividad a la situación. En la serie, cuando alienta a Walter a "pensar algo científico” para poder salir de allí, además de sugerir armar una batería, Jesse propone la creación de un "combustible para cohetes", un "robot", o la insólita posibilidad de convertir la camioneta en un vehículo de arena. Sin embargo, más allá de lo que los docentes caracterizaron como "ideas imaginativas, ideas infantiles", afirmaron que la ciencia aparece representada como un conocimiento que puede ayudarnos para resolver problemas prácticos: "Jesse ha visto que en la ciencia hay conocimiento técnico pero ahora también hay ideas", "Jesse le encuentra un sentido a ese conocimiento, un por qué, un para qué".

El análisis del fragmento IV aporta un nuevo sentido al conocimiento en relación con la utilización de la química para la fabricación de un artefacto explosivo: "Walter usa el conocimiento para vengarse, demuestra que tiene poder, usa el poder para engañar y quiere imponer miedo". Aquí surgieron los interrogantes respecto al uso del conocimiento: "la ciencia implica poder y el científico decide qué uso ético le da a la ciencia y al conocimiento”. Y entonces, la cuestión: “¿cuál es el límite?”. De este modo, la reflexión durante el plenario pudo girar en torno a preguntas tales como ¿cuál es la diferencia entre fabricar una batería, una bomba, metanfetaminas? Y, ¿cómo podrían trabajar estos temas en clase?

Finalmente, agregaron que la serie es conocida y difundida entre los estudiantes y que "pone en duda la ética que uno tiene" “¿por qué un profesor elige dejar su trabajo y dedicarse a hacer droga para tener más dinero? ¿Hay personas que defienden la fabricación de drogas? ¿Dónde hay 
un límite?” Y sobre la actitud de Walter como docente se cuestionaron: “¿por qué enseña si no quiere enseñar?”.

En resumen, el análisis de los fragmentos seleccionados puso en evidencia diferentes formas de concebir a la ciencia, al trabajo científico y a las personas que hacen ciencia, como así también, mostrar diferentes maneras de enseñarla. Al mismo tiempo, el debate estuvo permanentemente atravesado por los cuestionamientos éticos que llevaron a planteos personales y colectivos en torno a los roles docentes, al rol de los científicos y al complejo entramado social en donde todos estos procesos y situaciones tienen lugar.

\section{Conclusiones y Perspectivas}

Los resultados mostraron que el diseño de las actividades permitió evidenciar y describir las distintas concepciones de los docentes acerca de las modalidades de enseñanza, la imagen de ciencia y los usos del conocimiento científico, a partir de la articulación entre las miradas de la ética y la didáctica, en un trabajo de reflexión colectiva.

Consecuentemente, la construcción de este nuevo campo de investigación posibilitó la concreción de acciones que den muestra de esa génesis de una manera proactiva. En este sentido, el diseño de la propuesta didáctica, a partir de un cuidadoso entrelazamiento entre las cuestiones éticas y las ciencias experimentales, tanto en su faceta educativa como de investigación, es una muestra clara de la gran potencialidad del área planteada. Como ha quedado claro en el análisis y discusión de los resultados la propuesta promovió el debate y la reflexión sobre una serie de hechos con un amplio impacto social, mientras que la estrategia elegida demostró ser eficaz para acceder a una posibilidad de debate única. En este caso, la serie Breaking Bad puso en marcha un movimiento recursivo de análisis y reflexiones.

Desde un punto de vista educativo, el diseño permitió poner en acto estrategias para la inclusión del cine y las series para la enseñanza de las ciencias para su utilización en el espacio de clase con los estudiantes y también en capacitación a otros docentes. De este modo, quedó de manifiesto que la naturaleza del conocimiento profesional docente se construye a partir de un complejo entramado entre lo disciplinar, lo pedagógico, lo contextual y lo ético; y que por tanto, la formación y actualización permanente de los profesores para revisar sus prácticas docentes de acuerdo con los nuevos desafíos que involucran los cambios culturales y sociales resulta imprescindible.

Entre los aspectos encontrados más relevantes se encontraron el impacto social del conocimiento y la relevancia de las cuestiones sociocientíficas, al percibir el argumento de la serie sobre la producción y el consumo de drogas y la posible repercusión de esta temática en una clase de secundario, en contraposición a otros aspectos positivos como la fabricación de elementos para una mejor calidad de vida, el cuidado de la salud, de los otros y del ambiente.

Respecto de la imagen de la ciencia y las personas que se dedican a la ciencia surgieron concepciones diferentes de acuerdo con la situación considerada: la ciencia apareció como un conocimiento puntual, acabado, complejo, que da respuestas a problemas cotidianos; y también, como algo dañino, tanto a través de la fabricación de una droga, como de un artefacto explosivo destinado a destruir bienes materiales y a herir a otros seres humanos.

Esta última cuestión hace alusión directa al tema de los límites del conocimiento científico, que no son inherentes a la práctica misma. Las reglas morales interpelan a la actividad realizada de acuerdo con los códigos compartidos. La ética, por otra parte, nos permite sostener la tensión que implica poder pensar más allá de esos valores, y preguntarnos por los valores deseables y universalizables para los seres humanos. Así pues, la pregunta inicial por la fabricación de la droga (algo bueno o malo) permite adentrarnos en otro tipo de preguntas que no tengan respuestas dicotómicas. 
La relación entre la ética y la ciencia apareció durante la implementación del diseño, y sus manifestaciones evidenciaron la complejidad de esta interrelación: por un lado, los interrogantes acerca de los límites del conocimiento y su utilización, la responsabilidad del científico y del docente respecto de estos límites. La experiencia permitió además detectar un vacío en la formación ética de los profesores de ciencias que dio origen a nuevas preocupaciones entre los docentes participantes que amerita una profundización en el estudio de esta problemática a los fines de lograr una descripción más completa y contribuir a la capacitación docente en esta línea.

Así, este artículo intenta ser una contribución a este reclamo docente aportando nuevas herramientas conceptuales y legales para el tratamiento de problemas complejos.

\section{Referencias}

Adalian, J. (12 de agosto de 2013). Breaking Bad Returns to Its Biggest Ratings Ever. Vulture. Recuperado de http://www.vulture.com/2013/08/breaking-bad-returns-to-its-biggest-ratingsever.html [24 de septiembre de 2013].

Aguado, D. (2017). De Walter White a Heisenberg: El camino del (anti)héroe en la sociedad del riesgo. Anuario Electrónico de Estudios en Comunicación Social "Disertaciones”, 10(2), 208-224. DOI: 10.12804/revistas.urosario.edu.co/disertaciones/a.4666.

Almerich, G., Suárez, J. M., Orellana, N. y Díaz, M. I. (2010). La relación entre la integración de las tecnologías de la información y comunicación y su conocimiento. Revista de Investigación Educativa, 28(1), 31-50.

Astudillo, C.; Rivarosa, A. S. y Ortiz, F. (2012). La reflexión metacientífica a través del cine: un estudio sobre los saberes docentes. Revista Eureka sobre Enseñanza y Divulgación de las Ciencias, 9(3), 376-391.

Bell, R. L. y Lederman, N. G. (2003). Understandings of the Nature of Science and Decision Making on Science and Technology Based Issues. Science Education, 87, 352-377.

Bolívar, A. (2005). El lugar de la ética profesional en la formación universitaria. Revista Mexicana de Investigación Educativa, 10(24), 93-123.

Caena, F. (2011). Literature review Quality in Teachers' continuing professional development. Education and Training 2020. Thematic Working Group 'Professional Development of teachers'. European Commission.

Camacho, J. (2018). Identificación y caracterización de las creencias de docentes hombres y mujeres acerca de la relación ciencia-género en la educación científica. Estudios Pedagógicos, [S.l.], 43(3), 63-81.

Cambra, I.; Lorenzo, M. G. y Michel, J. (2018). Contribuciones del cine y la Psicología a la Enseñanza de la Física y otras ciencias naturales. El caso Copenhagen. Revista Enseñanza de la Física. En prensa.

Coll, C., Mauri, T. y Onrubia, J. (2008). Análisis de los usos reales de las TIC en contextos educativos formales: una aproximación sociocultural. Revista Electrónica de Investigación Educativa, 10(1). http://redie.uabc.mx/vol10no1/contenido-coll2.html [28 de octubre de 2017]

Cubero, R. (1989). Cómo trabajar con las ideas de los alumnos. Sevilla: Diada.

Dark, M. L. (2005). Using science fiction movies in introductory Physics. The Teacher Physics, 43, 463-465.

Darling, L. (2006). Constructing 21st-Century Teacher Education. Journal of Teacher Education, 57(3), 300-314.

Escudero, J. M. (2011). La formación continuada del profesorado, un tema crucial para la mejorar la educación. Madrid: Ministerio de Educación. 
Ferrareli, M. (2015). La textualidad des-bordada: transmedia y educación en la cultura digital. Lenguas vivas, 15(11), 8-18.

García-Borrás, F. (2006). Cuando los mundos chocan. Revista Eureka sobre Enseñanza y Divulgación de las Ciencias, 3(2), 268-286.

García-Borrás, F. (2008), Bienvenido Mister cine a la enseñanza de las ciencias. Revista Eureka sobre Enseñanza y Divulgación de las Ciencias, 6(1), 79-91.

García-Borrás, F. (2011), Las escenas cinematográficas: una herramienta para el estudio de las concepciones alternativas de física y química. Revista Eureka sobre Enseñanza y Divulgación de las Ciencias, 8(3), 291-311.

Gavidia, V. (2005). Los retos de la divulgación y enseñanza científica en el próximo futuro. Didáctica de las ciencias experimentales y sociales, 19, 91-102.

Gibbs, G. y Coffey, M. (2004). The impact of training of university teachers on their teaching skills, their approach to teaching and the approach to learning of their students. Active Learning in Higher Education, 5(1), 87-100. DOI: 10.1177/1469787404040463.

Grilli, J. (2015). Seven Pounds y Biología. Zoología y transplante de órganos en el epílogo del film, una experiencia en formación de docente. Didáctica de las ciencias experimentales y sociales, 29, 233-246.

Grilli, J. (2016). Cine de ciencia ficción y enseñanza de las ciencias. Dos escuelas paralelas que deben encontrarse en las aulas. Revista Eureka sobre Enseñanza y Divulgación de las Ciencias, 13(1), 137-148.

Guisasola, J. y Morentin, M. (2007). ¿Qué papel tienen las visitas escolares a los museos de ciencias en el aprendizaje de las ciencias? Una revisión de las investigaciones. Enseñanza de las Ciencias, 25(3), 401-414.

Hansen, D. (2001). Explorando el corazón moral de la enseñanza, Barcelona: IdeaBooks.

Jackson, P.; Boostrom, R. y Hansen, D. (2003). La vida moral en la escuela. Buenos Aires: Amorrortu.

Jenkins, H., Ford, S., y Green, J. (2013). Spreadable media: Creating value and meaning in a networked culture. New York: New York University Press.

Jiménez, R.; García, E.; Azcárate, P. y Navarrete, A. (2015). Dimensión ética de la sostenibilidad curricular en el sistema de evaluación de las aulas universitarias. El caso de la enseñanza aprendizaje de las Ciencias. Revista Eureka sobre Enseñanza y Divulgación de las Ciencias, 12(3), 536-549.

Law, N. y Chow, A. (2008). Teachers characteristics, contextual factors, and how these affect the pedagogical use of ICT. En Law, N.; Pelgrum, W.; Plomp, T. (Eds) Pedagogy and ICT use in schools around the world. Findings from the IEA SITES 2006 Study (pp.181-219). Nueva York: Springer.

López, M.L. y Nicolás, M. T. (2015) El análisis de series de televisión: construcción de un modelo interdisciplinario. ComHumanitas: Revista Científica de Comunicación, 6(1), 22-39.

Lorenzo, G., Farré, A., y Rossi, A. (2013). El uso de entornos virtuales en la capacitación docente de profesores universitarios. Enseñanza de las ciencias: revista de investigación y experiencias didácticas, Extra, 2014-2017.

Lorenzo, M. G. (2012). Los formadores de profesores: el desafío de enseñar enseñando. Profesorado: Revista de Currículum y Formación del profesorado, 16(2), 295-312.

Lorenzo, M. G. (2017). Enseñar y aprender ciencias. Nuevos escenarios para la interacción entre docentes y estudiantes. Educación y Educadores, 20(2), 249-263. DOI: 10.5294/edu.2017.20.2.5

Lorenzo, M. G. y Farré, A. S. (2016). La ciencia y la tecnología entre el bien y el mal. Un debate para la formación ciudadana. Aesthethika, International Journal on Subjectivity, Politics and the Arts, 12(3), 35-42. 
Mateus, J. C., y Chávez, R. (2014). ¿Educación en series? La integración de ficciones televisivas en el currículo universitario. En Blanco \& Negro, 5(1), 29-340.

Membiela, P. (1997). Una revisión del movimiento educativo ciencia-tecnología-sociedad. Enseñanza de las Ciencias, 15(1), 51- 57.

Michel, J.J. (2001). La ética en movimiento. Fundamentos en Humanidades. Facultad de Ciencias Humanas de San Luis, 1(2), 13-20.

Murillo, F.J. e Hidalgo, N. (2017). Hacia una Investigación Educativa Socialmente Comprometida. Revista Iberoamericana de Evaluación Educativa, 10(2), 5-8.

Nussbaum, L. (2017). Investigar con docentes. En E. Moore y M. Dooly (Eds), Enfoques cualitativos para la investigación en educación plurilingüe (pp. 23-45). Researchpublishing.net. DOI: 10.14705/rpnet.2017.emmd2016.620.

Ortega, M. L. (2002). Imágenes, conocimiento y educación. Reflexiones desde la historia de la representación visual en las ciencias. Tarbiya, 31, 11-38.

Palacios, S. (2007). El cine y la literatura de ciencia ficción como herramientas didácticas en la enseñanza de la física: una experiencia en el aula. Revista Eureka sobre Enseñanza y Divulgación de las Ciencias, 4(1), 106-122.

Perales, F. J., y Vílchez, J. M. (2005). The Teaching of Physics and Cartoons: Can they be interrelated in secondary education? International Journal of Science Education, 27(14), 1647-1670.

Petit, M. F. y Solbes, J. (2015). El cine de ciencia ficción en las clases de ciencias de enseñanza secundaria (I). Propuesta didáctica. Revista Eureka sobre Enseñanza y Divulgación de las Ciencias, 12(2), 311-327.

Petit, M.F. y Solbes, J. (2016). El cine de ciencia ficción en las clases de ciencias de enseñanza secundaria (II). Análisis de películas. Revista Eureka sobre Enseñanza y Divulgación de las Ciencias, 13(1), 176-191.

Pro-Bueno, A. D. y Ezquerra, A. (2005). ¿Qué ciencia ve nuestra sociedad? Alambique: Didáctica de las Ciencias Experimentales, 12(43), 37-48.

Pujalte, A., Adúriz-Bravo, A. y Porro, S. (2015). Las imágenes de ciencia en profesoras y profesores de Biología: Entre lo que se dice y lo que se hace, Revista Boletín Biológica, 9(33), 5-10.

Ruiz, J. J. y Solbes, J. (2013). Debates sobre cuestiones sociocientíficas. Una herramienta para aprender física y química. Textos de Didáctica de la Lengua y de la Literatura, 64, 32-39.

Ryan, M. (2012). 'Breaking Bad': Five Reasons It's One Of TV's All-Time Greats. Huffington Post. Recuperado de https://www.huffingtonpost.com/maureen-ryan/breaking-bad-greatestshow_b_1665640.html [24 de septiembre de 2013].

Shulman, L. S. (1986). Those who understand: Knowledge growth in teaching. Educational researcher, 15(2), 4-14.

Sierra, C. E. (2007). Fortalezas epistemológicas y axiológicas de la ciencia ficción: un Potosí pedagógico mal aprovechado en la enseñanza y divulgación de las ciencias. Revista Eureka para la Enseñanza y Divulgación de las Ciencias, 4(1), 87-105.

Solbes, J. (1999). Los valores en la enseñanza de las ciencias. Alambique, 22, 1-7.

Solbes, J. y Torres, N. (2014). Aspectos convergentes del pensamiento crítico y las cuestiones sociocientíficas. Góndola, Enseñanza y Aprendizaje de las Ciencias, 9(1), 1-13.

Solbes, J. y Torres, N. (2015). Alternativas para reflexionar aspectos críticos de la ciencia en el aula. Revista científica, 22, 1-17.

Solbes, J. y Traver, M. J. (1996). La utilización de la historia de las ciencias en la enseñanza de la física y la química. Enseñanza de las ciencias, 14(1), 103-112.

Solbes, J. y Traver, M. (2001). Resultados obtenidos introduciendo historia de la ciencia en las clases de física y química: mejora de la imagen de la ciencia y desarrollo de actitudes positivas. Enseñanza de las ciencias, 19(1), 151-162. 
Solbes, J. y Vilches, A. (1989). Interacciones ciencia/técnica/sociedad: un instrumento de cambio actitudinal. Enseñanza de las Ciencias, 7(l), 14-20.

Solbes, J. y Vilches, A. (2004). Papel de las relaciones entre ciencia, tecnología, sociedad y ambiente en la formación ciudadana. Enseñanza de las Ciencias, 22(3), 337 -348.

Solbes, J., Monserrat, R. y Furió, C. (2007). El desinterés del alumnado hacia el aprendizaje de la ciencia: implicaciones en su enseñanza. Didáctica de las Ciencias Experimentales y Sociales, 21, 91-117.

Taylor, S. J. y Bodgan, R. (1992). Introducción a los métodos cualitativos de la investigación. Barcelona: Paidós.

Tearle, P. (2003). ICT implementation: What makes the difference? British Journal of Educational Technology, 34(5), 567-584.

Timperley, H. y Alton-Lee, A. (2008). Reframing teacher professional learning: An alternative Policy Approach to strengthening valued outcomes for diverse learners. Review of Research in Education, 32, 1-45.

Torre, L., y García, S. (2014). La cristalografía en el cine. Anales de Química, 110(1), 72-77.

Vieira, R. y Nascimento, S. (2007). A argumentação no discurso de um professor e seus estudantes sobre um tópico de mecânica newtoniana. Caderno Brasileiro de Ensino de Física, 24(2), 174-193.

Wei, R., Darling, L. y Adamson, F. (2010). Professional Development in EEUU: Trends and Challenges. Dallas, TX. National Staff. Development Council. National Staff Development Council.

Zúñiga, A., Leiton, R. y Naranjo, J. (2014). Del sistema educativo tradicional hacia la formación por competencias: Una mirada a los procesos de enseñanza aprendizaje de las ciencias en la educación secundaria de Mendoza Argentina y San José de Costa Rica. Revista Eureka sobre Enseñanza y Divulgación de las Ciencias, 11(2), 145-159. DOI: 10498/15972

CÓMO CITAR ESTE ARTÍ́CULO

Cambra, I. y Lorenzo, M. G. (2018). Entrelazando la Ética con las Ciencias Experimentales: una propuesta didáctica para la capacitación de profesores con la serie Breaking Bad. Didáctica de las ciencias experimentales y sociales, 34, 105-122. DOI: 10.7203/DCES.34.11478. 
